Z. Epileptol. $2021 \cdot 34: 403-408$ https://doi.org/10.1007/s10309-021-00438-9

Angenommen: 16. August 2021

Online publiziert: 7. Oktober 2021

๑) Der/die Autor(en) 2021

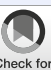

\title{
Akut symptomatische Anfälle und die Abgrenzung zum Status epilepticus auf der Intensivstation bei Kindern und Erwachsenen
}

Markus Leitinger

Universitätsklinik für Neurologie Salzburg, Christian-Doppler-Klinik, Paracelsus Medizinische Universität Salzburg, Affiliated Member of the European Reference Network, Salzburg, Österreich

\section{Zusammenfassung}

Akut symptomatische Anfälle (ASA) finden sich häufig an pädiatrischen und neurologischen Intensivstationen. Während bei Vorliegen einer fassbaren Semiologie die Erkennung klinisch gelingt, bedarf es für elektrographische Anfälle präziser Kriterien, welche von der Amerikanischen Klinischen Neurophysiologischen Gesellschaft (ACNS) kürzlich in einer aktualisierten Form publiziert wurden. Neue Aspekte sind hierbei die Beschreibung von kurzen potenziell iktalen rhythmischen Entladungen („brief potentially ictal rhythmic discharges" [BIRDs]) sowie die Beschreibung des IktalInteriktalen-Kontinuums (IIC). Die Interpretation der elektroenzephalographischen (EEG) Kriterien erfolgt im Idealfall in einem interdisziplinären Zugang zwischen der Neurophysiologie und der Intensivmedizin. Die Korrelation von Anfallslast mit Verschlechterung des neurologischen Outcomes ist in Studien an pädiatrischen bzw. neurologischen Intensivstationen belegt.

\section{Schlüsselwörter}

BIRDs („,brief potentially ictal rhythmic discharges“) · IIC (Iktal-Interiktales-Kontinuum) .

Anfallslast · Definition · ACNS (Amerikanische Klinische Neurophysiologische Gesellschaft)

Epileptische Anfälle sind Ausdruck einer signifikanten Hirnfunktionsstörung und bedürfen der raschen neurologischen Abklärung. Auf der Intensivstation und in der Notaufnahme kommt Patientinnen und Patienten mit epileptischen Anfällen ohne vorbestehende Epilepsie eine besondere Bedeutung zu. Einerseits ist zunächst unklar, ob sich weitere Anfälle ereignen werden bzw. sich ein Status epilepticus entwickeln wird, andererseits muss möglichst rasch die Ursache identifiziert werden, da viele akute neurologische Erkrankungen wie zerebrale Ischämie oder bakterielle bzw. virale Meningoenzephalitis selbst eine zeitkritische Therapie erfordern. Die Ursache oder die Auswirkungen der epileptischen Aktivität können zur Aufnahme an neurologische oder pädiatrische Intensivstationen führen, darüber hinaus treten epileptische Anfälle auch erstmals bei intensivmedizinisch behandelten Patientinnen und Patienten auf. Dieser Artikel legt den Schwerpunkt auf die Diagnostik von akut symptomatischen Anfällen bzw. deren Abgrenzung vom Status epilepticus.

\section{Abgrenzung der akut sympto- matischen Anfälle zum Status epilepticus}

Akut symptomatische Anfälle (ASA) unterscheiden sich vom Status epilepticus (SE) durch 2 wesentliche Kriterien: erstens das Zeitkriterium, das für bilateral tonisch-klonische Entäußerungen 5 min beträgt und bei längerer Anfallsaktivität das Vorliegen eines "konvulsiven“ Status epilepticus determiniert. Für die anderen Formen des "Status epilepticus mit prominent motori- 


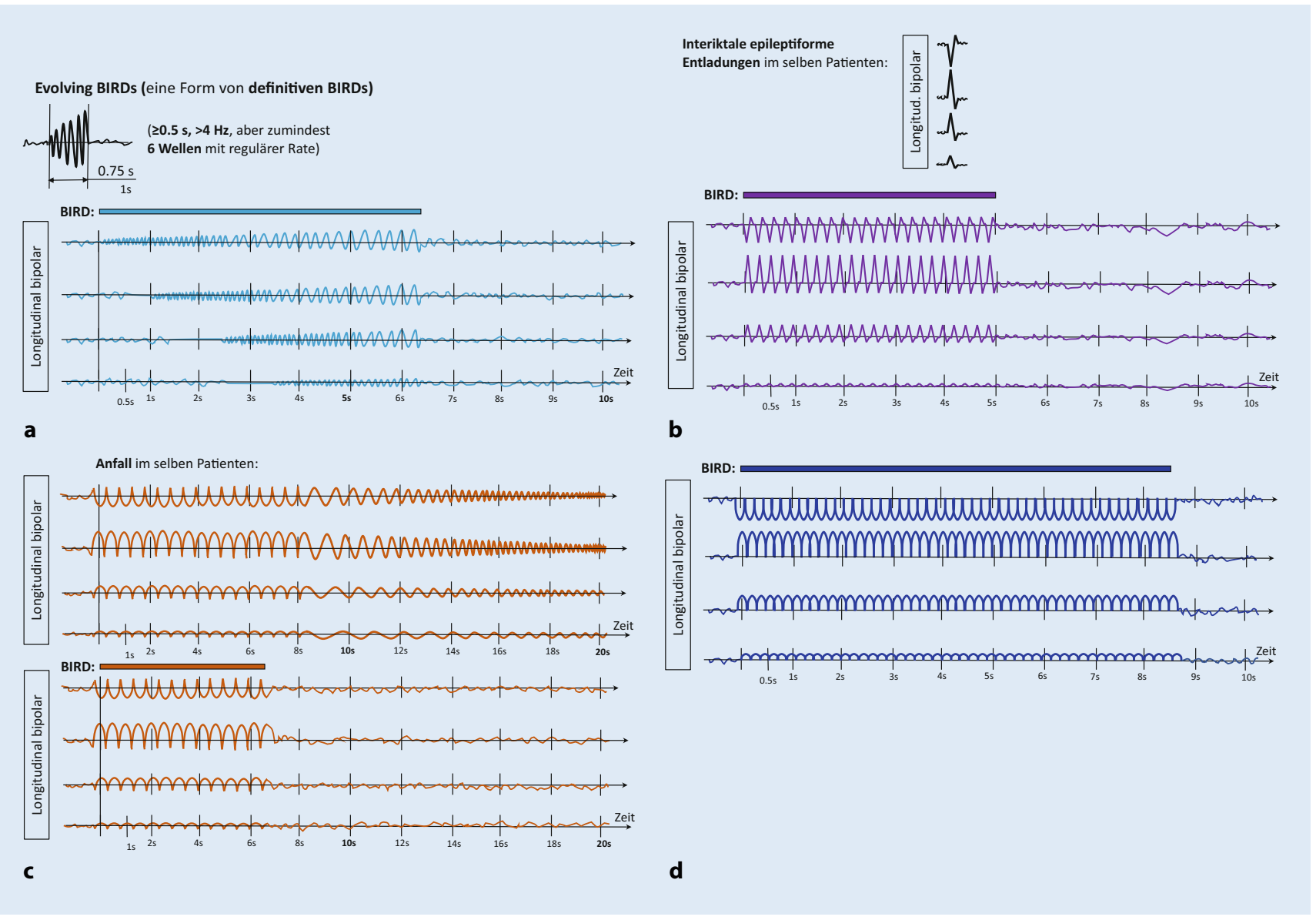

Abb. 1 ム Kurze potenziell iktale rhythmische Entladungen („brief potentially ictal rhythmic discharges“) BIRDs. Fokal (einschließlich Lateralisiert [L], Bilateral unabhängig - independent [BI], Einseitig unabhängig [UI], oder Multifokal [Mf]) oder generalisierte rhythmische Aktivität $>4 \mathrm{~Hz}$ (mit zumindest 6 Wellen mit einer regulären Rate) für $\geq 0.5$ bis $<10$ s andauernd. 1. nicht übereinstimmend mit einem bekannten normalen Muster oder einer gutartigen Variante, 2. nicht Teil eines Burst-Suppression- oder Burst-Attenuation-Musters, 3. ohne definitives klinisches Korrelat, 4. und das zumindest eines der folgenden Eigenschaften aufweist: a Evolving BIRDs (eine Form von definitiven BIRDs), b, c Gleiche Morphologie und Lokalisation wie interiktale epileptiforme Entladungen oder Anfälle im selben Patienten (definitive BIRDs), d Scharf konturiert aber ohne Kriterien (a, b oder c) (mögliche bzw. possible BIRDs). (Mit freundl. Genehmigung des Verlages Wolters Kluwer Health, Inc. gemäß Hirsch et al. 2021 [5])

schen Symptomen" (z. B. fokal motorisch), gilt ebenfalls die 5 Minuten Grenze [1]. Analog ist eine Dauer von 10 min für fokal bewusst bzw. nicht bewusst erlebte Anfallsaktivität sowie Absencen zur Klassifikation als Status epilepticus anzuwenden [1]. Hinsichtlich der bisherigen Studienlage wird insbesondere der Zeitraum zwischen $5 \mathrm{~min}$ und 30 min je nach Definition in den verschiedenen Studien als Anfall oder als Status epilepticus gewertet [2]. In einer indischen Studie war der Anteil der Kinder mit Anfallsaktivität unter 30 min etwas größer als jener über $30 \mathrm{~min}$ [3]. Aus wissenschaftlicher Sicht sollten alle Zeiträume näher untersucht werden, beispielsweise hinsichtlich des Ansprechens auf Anti-Anfalls-Medikation, weshalb möglichst frühe Formen ebenfalls eingeschlossen werden sollen. Zweitens stellt die definierende akut symptomatische Genese bei ASA nur eine von mehreren ätiologischen Gruppen dar, während der Status epilepticus zusätzlich die zurückliegenden („remote“) und die voranschreitenden Ursachen („progressive“), die „definierten elektroklinischen Syndrome" und die ungeklärten Ätiologien („kryptogen") beinhaltet [1].

\section{Klinische Präsentation}

Die Semiologie der akut symptomatischen Anfälle entspricht grundsätzlich dem gesamten Spektrum an Anfällen [4]. Liegen jedoch keine klinisch fassbaren Anfallszeichen oder allenfalls sehr subtile mo- torische Phänomene (diskrete Zuckungen perioral bzw. periorbital, Nystagmus, forcierte iktale Blickwendung) vor, so wird dies im Falle eines Status epilepticus als "nichtkonvulsiv" zusammengefasst [1]. Im Gegensatz dazu bildet die Anfallsklassifikation der Internationale Liga gegen Epilepsie (ILAE) von Fisher et al. 2017 elektroenzephalographisch iktale bzw. subklinische Muster nicht ab [4]. Die nichtkonvulsiven Formen können zwar einen klinischen Verdacht ergeben, bedürfen jedoch zur sicheren Diagnosestellung eines EEGNachweises, wobei hierbei im Idealfall ein kontinuierliches EEG (cEEG) eine bessere zeitliche Abdeckung und damit einhergehende Sensitivität und Quantifizierbarkeit der Veränderungen erlaubt. 


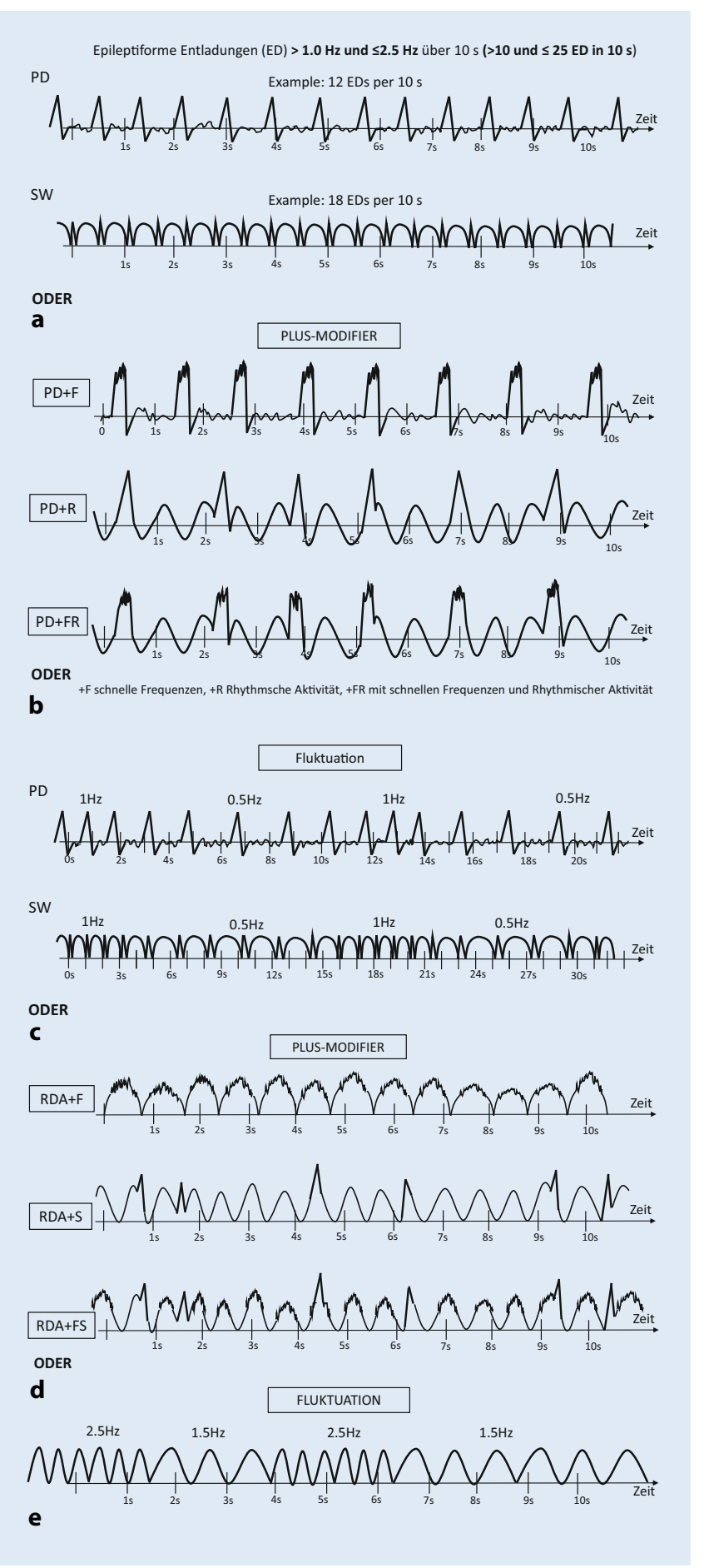

Abb. $2 \Delta$ Iktal-Interiktales Continuum (IIC). Diagnostische EEG-Kriterien ( $+F$, schnelle Aktivität; $+R$, rhythmische Aktivität; $+S$, „sharp waves“ oder Spikes oder scharf konturiert). Qualifiziert sich nicht als elektrographischer Anfall oder elektrographischer Status epilepticus, kann allerdings bei folgenden Merkmalen in Erwägung gezogen werden: a Jegliches Muster aus Periodische Entladungen (PD) oder Spike and Waves bzw. Sharp and Waves (SW), dessen Durchschnittsfrequenz von $>1.0 \mathrm{~Hz}$ bis $<2.5 \mathrm{~Hz}$ beträgt und über $10 \mathrm{~s}$ dauert ( $>10$ und $<25$ Entladungen in $10 \mathrm{~s}$ ), b, c Jegliches PD oder SW Muster mit einer Durchschnittsfrequenz von $\geq 0.5 \mathrm{~Hz}$ bis $\leq 1.0 \mathrm{~Hz}$ über 10 Sekunden $(\geq 5$ und $\leq 10$ Entladungen in $10 \mathrm{~s}$ ), UND einen Plus-Modifier oder Fluktuation (Fluktuation gemäß der Definition der ACNS) aufweist, $\mathbf{d}$, e Jegliche lateralisierte Rhythmsiche Delta Aktivität, RDA (LRDA, BIRDA, UIRDA, MfRDA) mit einer Durchschnittsfrequenz von $>1 \mathrm{~Hz}$ für $\geq 10 \mathrm{~s}$ (zumindest 10 Wellen in $10 \mathrm{~s}$ ) mit einem Plus-Modifier oder Fluktuation (Fluktuation gemäß ACNS). (Mit freundl. Genehmigung des Verlages Wolters Kluwer Health, Inc. gemäß Hirsch et al. 2021 [5])

\section{EEG-Kriterien}

Die rezenteste Veröffentlichung der Amerikanischen Klinischen Neurophysiologischen Gesellschaft (ACNS) fordert für elektrographische Anfälle epileptiforme Entladungen über $2,5 \mathrm{~Hz}$ (bzw. 25/10s) oder jegliches Muster mit definitiver Evolution für jeweils mindestens $10 \mathrm{~s}$, wobei eine "Evolution" gemäß ACNS (1) in der Frequenz, (2) in der Lokalisation oder (3) in der Morphologie auftreten kann [5]. Evidenz für das Frequenzkriterium $2,5 \mathrm{~Hz}$ stammt von intrakraniellen multimodalen Untersuchungen, die bei periodischen epileptiformen Entladungen einen Abfall des Gewebesauerstoffpartialdruckes als Ausdruck der metabolen Erschöpfung nachweisen konnten [6]. Ein elektrographischer Status epilepticus besteht gemäß ACNS bei elektrographischer Anfallsaktivität für mindestens 10 min oder bei mindestens $20 \%$ einer 60 -min-EEGAbleitung [5]. Ein elektroklinischer Anfall zeigt entweder ein definitives klinisches Korrelat zeitgebunden zu einem Muster jeglicher Dauer (also auch unter 10 s Dauer) oder eine Verbesserung des EEGs und der klinischen Performance auf ein parenteral typischerweise intravenös verabreichtes Ani-Anfalls-Medikament (AAM) [5]. Ein elektroklinischer Status epilepticus stellt eine elektroklinische Anfallsaktivität für mindestens 10 min oder mindestens $20 \%$ einer 60-min-Ableitung oder mindestens 5 min kontinuierlicher bilateral tonisch klonischer Aktivität dar [5].

Anfallssuspekte Muster einer rhythmischen Aktivität von mehr als $4 \mathrm{~Hz}$ ohne klinisches Korrelat in der Dauer von mindestens einer halben Sekunde und weniger als $10 \mathrm{~s}$ können sich als kurze potenziell iktale rhythmische Entladungen („,brief potentially ictal rhythmic discharges" [BIRDs]) qualifizieren ([5, 7, 8]; - Abb. 1). Im Detail werden "definitive BIRDs" mit Evolutionsmuster (- Abb. 1a) oder gleicher Morphologie und Lokalisation wie interiktale Entladungen ( $\bullet$ Abb. 1b) oder Anfälle (• Abb. 1c) von "möglichen BIRDs" mit scharfer Kontur ohne die vorgenannten Kriterien ( $\bullet$ Abb. 1d) abgegrenzt [5]. Der exakte Stellenwert von BIRDs ist noch Gegenstand der Forschung, es gibt jedoch Hinweise darauf, dass BIRDs wie Anfäl- 
le Hinweise auf die Anfallsursprungszone geben [8].

Im Intensivbereich werden oft höhergradig pathologische EEGs abgeleitet mit der Notwendigkeit, Anfalls- oder Statusaktivität von anderweitigen z. B. enzephalopathischen Veränderungen abzugrenzen.

\section{॥ Der klinische Kontext ist entscheidend}

Das „Iktale-Interiktale Continuum“ (IIC) stellt den Übergangsbereich zwischen eindeutig iktaler Aktivität und andererseits eindeutig nichtiktaler Aktivität als Extrempunkte eines Spektrums dar und wird gemäß ACNS als Synonym für „möglichen elektrographischen Anfall" bzw. "möglichen elektrographischen Status epilepticus" betrachtet [5]. Kurz zusammengefasst werden Frequenzbereiche von Periodischen Entladungen (PD), "sharp waves" oder Lateralisierter Rhythmischer Delta-Aktivität (LRDA) festgelegt, wobei in bestimmten langsameren Bereichen Zusatzbedingungen wie „Plus-

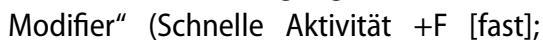
Rhythmische Aktivität $+R$; "sharp waves" oder Spikes oder scharf konturiert $+S$ ) oder Fluktuation erfüllt sein müssen und sich nicht für elektrographische Anfälle oder elektrographischen Status epilepticus qualifizieren dürfen (- Abb. 2; [5]). Als „möglicher elektroklinischer Anfall bzw. Status epilepticus" wird ein rhythmisches oder periodisches Muster angesehen, das sich als IIC mit oben genannten Zeitkriterien (mindestens 10 Minuten oder 20\% einer 60 Minuten Ableitung) qualifiziert und auf parenterale Anti-Anfalls-Medikamente eine EEG-Verbesserung ohne Verbesserung der klinischen Performance zeigt [5]. Dies soll die probatorische Gabe von Anfallsmedikamenten zu Diagnosezwecken anregen. Einschränkend ist jedoch festzuhalten, dass Parameter wie "Verbesserung im EEG" oder "Verbesserung der klinischen Performance" bislang weder durch Kriterien noch hinsichtlich eines standardisierten Untersuchungszeitraumes konsensuell festgelegt sind. Darüber hinaus ist die Verwendung des Terminus „möglicher Status epilepticus" im klinischen Alltag nicht unproblematisch, da in der Kommunikation unklar bleibt, ob nun eine Statustherapie indiziert ist oder nicht.

Die von den ACNS empfohlene Interpretation des IIC im gesamten klinischen Kontext sollte nach Ansicht des Verfassers von Neurophysiologinnen bzw. Neurophysiologen und pädiatrischen bzw. neurologischen Intensivmedizinerinnen bzw. Intensivmedizinern gemeinsam erfolgen [5]. Die Gefahr besteht nach Ansicht des Verfassers darin, dass man diesen letzten Schritt der individuellen Reflexion im klinischen Kontext nicht durchführt und ein „möglicher Status epilepticus" entweder niemals als "Status epilepticus" interpretiert wird und somit aufgrund der falsch negativen Diagnose eine Untertherapie bewirkt, oder immer mit "Status epilepticus" gleichgesetzt wird, was in falsch positiven Diagnosen eines SE und damit in einer etwaigen Übertherapie resultiert [9].

\section{Anfallslast}

Bei Anfällen, insbesondere elektrographischen Anfällen, besteht die Frage, ob diese nur Ausdruck der Gewebeschädigung und somit ein Epi- bzw. Begleitphänomen darstellen, oder ob sie selbst dem Gehirn Schaden zufügen können. Im ersteren Fall stellt eine Medikation eine zusätzliche unnötige Belastung der Patientinnen und Patienten dar, im letzteren Fall ist eine rasche Unterbrechung des epileptischen Geschehens indiziert.

Für den pädiatrischen Bereich ist hierzu die Arbeit von Payne et al. 2014 zu nennen, in der anhand von 259 Kindern im medianen Alter von 2,2 Jahren (Interquartilenbereich 0,3 Tage bis 9,7 Jahre) eine „dosisabhängige" Schädigung in Form eines schlechteren Ergebniswertes auf der Paediatric Cerebral Performance Category(PCPC)-Skala bei Entlassung nachgewiesen werden konnte [10]. Die PCPC-Skala enthält die Kategorien „normal“, „milde“, „moderate" und „schwere Beeinträchtigung", "Koma oder vegetativer Zustand" sowie "Hirntod" [11]. Als "Schwellenwert" für eine definitive ZNS-Schädigung wurden $20 \%$ einer elektroenzephalographischen Ableitung identifiziert, somit 12 min einer Ableitungsstunde, was in die ACNS-Kriterien Eingang gefunden hat $[5,10]$. Andererseits konnte eine Odds Ratio für neurologische Verschlechterung von 1,13 für jede $1 \%$ Anstieg der maximalen stündlichen Anfallslast gezeigt werden, wobei diese Dosis-Wirkungs-Beziehung insbesondere für Anfälle ohne nachweisbare Ätiologie und für Anfälle bei Systemerkrankungen galt, während die Gruppe mit akuter Hirnschädigung mit akut symptomatischen Anfällen eine hohe A-priori-Wahrscheinlichkeit für neurologische Verschlechterung aufwies [10]. Die maximale stündliche Anfallslast ist ein im klinischen Alltag praktischer Parameter, da er im Gegensatz zur Gesamtanfallslast sofort klar ersichtlich ist mit der Möglichkeit einer Therapieanpassung [10]. Außerdem ist sehr gut nachvollziehbar, dass die Anfallslast in einer bestimmten Zeiteinheit für das Gehirn relevant ist, da dies ja die metabolen Reserven herausfordert [10]. Einen ausgezeichneten Überblick über die pädiatrische Studienlage bezüglich der Anfallslast sowie die technischen Herausforderungen hierbei geben Ganesan und Hahn 2019 [12].

\section{॥ Steigende Anfallslast als Ursache dosisabhängiger neurologischer Verschlechterung}

De Marchis et al. zeigten mittels einer multivariaten Analyse an 50 erwachsenen Patientinnen und Patienten mit Anfällen (in einer Population aus 402 Personen mit Subarachnoidalblutung), dass 3 Monate nach Entlassung kognitive und funktionelle Defizite bestanden, deren Ausmaß mit der Anfallslast korrelierte [13]. Jede Stunde mit Anfällen führte zu einer Odds Ratio von 1,10 für schlechteres Outcome [13]. In einer weiteren Arbeit über Personen mit einer Subarachnoidalblutung wurde der Beitrag nichtkonvulsiver Anfälle als Bindeglied zwischen Entzündung und Outcome erarbeitet [14]. Eine Gruppe von 6 Personen mit Schädel-Hirn-Trauma (SHT) und Anfällen zeigte eine signifikante Atrophie der Hippocampi im Vergleich zu einer alters- und Glasgow-Coma-Scale-gematchten Vergleichsgruppe mit SHT jedoch ohne Anfälle [15].

\section{Epidemiologie}

In der Rochester-Studie betrug im Zeitraum 1955 bis 1984 die Inzidenz für ASA 39,0/100.000 Personenjahre (altersadjus- 
tiert für Männer 52,0 und für Frauen 29,5), wobei alle Altersgruppen eingeschlossen waren [16]. Das kumulative Risiko, bis zum 80. Lebensjahr einen akut symptomatischen Anfall zu erleiden, betrug 3,6\% [16]. Die Hauptursachen waren Schädel-Hirn-Trauma (16\%), zerebrovaskuläre Erkrankungen (16\%), Zentralnervensystem(ZNS)-Infektionen (15\%), Medikamentenentzug (14\%) und metabole Ursachen (9\%) [16]. Das Spektrum der Ätiologien variiert je nach Altersgruppen mit einem Maximum für Infektionen im Kindesalter, Medikamentenentzug im Erwachsenenalter und zerebrovaskulären Ursachen im Senium [16].

Neurologische Erkrankungen tragen global zu 16\% der Aufnahmen auf eine pädiatrische Intensive Care Unit (PICU) bei [17]. In einer indischen Studie an 157 Kindern im Alter von 1 Monat bis 16 Jahren erlitten $64 \%$ ASA, davon waren die häufigsten Ursachen ZNS-Infektionen (45\%), SHT (13\%), metabolisch (12\%), febril (7\%), und hypokalziämisch (6\%), hypertensiv (4\%), hypoxisch (4\%), Shuntblockade (3\%), hypoglykämisch (2\%) und Schlaganfall (2\%) [3]. Ein Alter unter 2 Jahren, hoher Pediatric Early Warning Sign(PEWS)-Score bei Einlieferung, prolongierte bzw. wiederkehrende Anfälle, ZNSInfektionen (insbesondere tuberkulöse Meningitis) und der Bedarf an mehreren Anfallsmedikamenten sowie Respiratorbzw. Kreislaufunterstützung waren signifikante Risikofaktoren für schlechtes Outcome [3]. Bei Anwendung des PEWS werden 0 bis 3 Punkte in den Domänen "Verhalten", "kardiovaskulär" und "respiratorisch" vergeben, wobei eine höhere Punktezahl ein erhöhtes Risiko ausdrückt $[18,19]$. ASA betrugen $53,3 \%$ in einer PICU in der Türkei [20].

\section{Zusammenfassung}

Studien ergeben Hinweise darauf, dass Anfälle eine dosisabhängige negative Auswirkung auf das Outcome haben, wobei hier ein kumulativer Effekt von Anfällen und Status epilepticus besteht. Die exakte Diagnostik wird durch Kriterien insbesondere der Amerikanischen Klinischen Neurophysiologischen Gesellschaft (ACNS) ermöglicht, ebenso die Evaluierung der Muster des IIC im gesamten klinischen Kontext.
ASA haben ein hohes a-priori-Risiko für neurologische Verschlechterung und bedürfen der raschen Ursachenabklärung zur Einleitung einer spezifischen Therapie.

\section{Fazit für die Praxis}

Die Ätiologie von Anfällen ist auf der Intensivstation bzw. in der Notaufnahme nicht sofort erkennbar, weshalb vorerst von einem akut symptomatischen Anfall auszugehen ist und eine rasche und akribische Ursachensuche eingeleitet werden muss, damit zeitkritische Therapien frühestmöglich initiiert werden. Studien belegen die Verschlechterung des neurologischen Outcomes mit zunehmender Anfallslast bzw. maximaler stündlicher Anfallslast bei Kindern und Erwachsenen. Bei der Diagnose elektrographischer bzw. elektroklinischer Anfälle bzw. Status epilepticus ist die neue Nomenklatur der ACNS sehr hilfreich, wobei sich in der Publikation zahlreiche Schemata bzw. Abbildungen zur optimalen Illustration befinden. Die Kriterien benötigen zu jedem Zeitpunkt die Interpretation im gesamten klinischen Kontext, idealerweise im Rahmen der interdisziplinären Zusammenarbeit von Kolleginnen und Kollegen der Neurophysiologie sowie der pädiatrischen bzw. neurologischen Intensivmedizin.

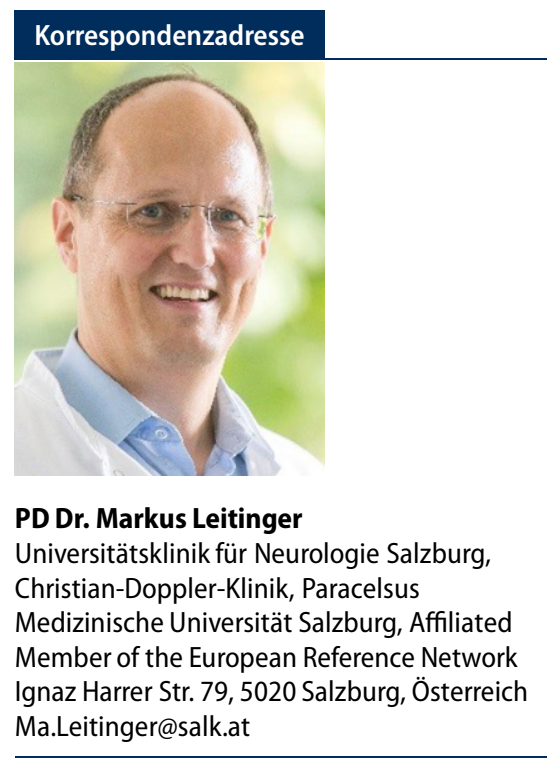

Funding. Open access funding provided by Paracelsus Medical University.

\section{Einhaltung ethischer Richtlinien}

Interessenkonflikt. Markus Leitinger berichtet über eine kongressbezogene Reiseunterstützung von UCB
Pharma und ein Vortragshonorar von Eisai, alle ohne Bezug zur vorliegenden Arbeit.

Für diesen Beitrag wurden vom Autor keine Studien an Menschen oder Tieren durchgeführt. Für die aufgeführten Studien gelten die jeweils dort angegebenen ethischen Richtlinien.

Open Access. Dieser Artikel wird unter der Creative Commons Namensnennung 4.0 International Lizenz veröffentlicht, welche die Nutzung, Vervielfältigung, Bearbeitung, Verbreitung und Wiedergabe in jeglichem Medium und Format erlaubt, sofern Sie den/die ursprünglichen Autor(en) und die Quelle ordnungsgemäß nennen, einen Link zur Creative Commons Lizenz beifügen und angeben, ob Änderungen vorgenommen wurden.

Die in diesem Artikel enthaltenen Bilder und sonstiges Drittmaterial unterliegen ebenfalls der genannten Creative Commons Lizenz, sofern sich aus der Abbildungslegende nichts anderes ergibt. Sofern das betreffende Material nicht unter der genannten Creative Commons Lizenz steht und die betreffende Handlung nicht nach gesetzlichen Vorschriften erlaubt ist, ist für die oben aufgeführten Weiterverwendungen des $\mathrm{Ma}$ terials die Einwilligung des jeweiligen Rechteinhabers einzuholen.

Weitere Details zur Lizenz entnehmen Sie bitte der Lizenzinformation auf http://creativecommons.org/ licenses/by/4.0/deed.de.

\section{Literatur}

1. Trinka $E$, Cock $H$, Hesdorffer D, Rossetti AO, Scheffer IE, Shinnar S, Shorvon S, Lowenstein DH (2015) A definition and classification of status epilepticus-report of the ILAE Task Force on Classification of Status Epilepticus. Epilepsia 56(10):1515-1523. https://doi.org/10.1111/epi. 13121

2. Leitinger M, Trinka E, Zimmermann G, Granbichler CA, Kobulashvili T, Siebert U (2020) Epidemiology of status epilepticus in adults: apples, pears, and oranges - a critical review. Epilepsy Behav 103(Pt A):106720. https://doi.org/10.1016/ j.yebeh.2019.106720

3. Amonkar P, N R, Gavhane J (2020) A study of critically ill children presenting with seizures regardless of seizure duration admitted in the PICU of a tertiary hospital in India. Epilepsy Behav Rep 14:100382. https://doi.org/10.1016/j.ebr.2020. 100382

4. Fisher RS, Cross JH, French JA, Higurashi N, Hirsch E, Jansen FE, Lagae L, Moshé SL, Peltola J, Roulet Perez E, Scheffer IE, Zuberi SM (2017) Operational classification of seizure types by the International League AgainstEpilepsy: position paper of the ILAE Commission for Classification and Terminology. Epilepsia 58(4):522-530. https://doi.org/10.1111/ epi.13670

5. Hirsch LJ, Fong MWK, Leitinger $M$, LaRoche SM, Beniczky S, Abend NS, Lee JW, WusthoffCJ, Hahn CD, Westover MB, Gerard EE, Herman ST, Haider HA, Osman G, Rodriguez-Ruiz A, Maciel CB, Gilmore EJ, Fernandez A, Rosenthal ES, Claassen J, Husain AM, Yoo JY, So EL, Kaplan PW, Nuwer MR, van Putten M, Sutter R, Drislane FW, Trinka E, Gaspard N (2021) American Clinical Neurophysiology Society's standardized critical care EEG terminology: 2021 
version. J Clin Neurophysiol 38(1):1-29. https:// doi.org/10.1097/WNP.0000000000000806

6. Witsch J, Frey HP, Schmidt JM, Velazquez A, Falo CM, Reznik M, Roh D, Agarwal S, Park S, Connolly ES, Claassen J (2017) Electroencephalographic periodic discharges and frequency-dependent brain tissue hypoxia in acute brain injury. JAMA Neurol 74(3):301-309. https://doi.org/10.1001/ jamaneurol.2016.5325

7. Yoo JY, Rampal N, Petroff OA, Hirsch LJ, Gaspard N (2014) Brief potentially ictal rhythmic discharges in critically ill adults. JAMA Neurol 71(4):454-462. https://doi.org/10.1001/jamaneurol.2013.6238

8. Yoo JY, Jetté N, Kwon CS, Young J, Marcuse LV, Fields MC, Gaspard N, Hirsch LJ (2021) Brief potentially ictal rhythmic discharges and paroxysmal fast activity as scalp electroencephalographic biomarkers of seizure activity and seizure onset zone. Epilepsia 62(3):742-751. https://doi.org/10. 1111/epi.16822

9. Leitinger M, Beniczky S, Rohracher A, Gardella E, Kalss G, Qerama E, Höfler J, Hess Lindberg-Larsen A Kuchukhidze G, Dobesberger J, Langthaler PB, Trinka E (2015) Salzburg consensus criteria for non-convulsive status epilepticus - approach to clinical application. Epilepsy Behav 49:158-163. https://doi.org/10.1016/j.yebeh.2015.05.007

10. Payne ET, Zhao XY, Frndova H, McBain K, Sharma R, Hutchison JS, Hahn CD (2014) Seizure burden is independently associated with short term outcome in critically ill children. Brain $137(\mathrm{Pt}$ 5):1429-1438. https://doi.org/10.1093/brain/ awu042

11. Fiser DH, Long N, Roberson PK, Hefley G, Zolten K, Brodie-Fowler M (2000) Relationship of pediatric overall performance category and pediatric cerebral performance category scores at pediatric intensive care unit discharge with outcome measures collected at hospital discharge and 1- and 6-month follow-up assessments. Crit Care Med 28(7):2616-2620. https://doi.org/10.1097/ 00003246-200007000-00072

12. Lalgudi Ganesan S, Hahn CD (2019) Electrographic seizure burden and outcomes following pediatric status epilepticus. Epilepsy Behav 101(Pt B):106409. https://doi.org/10.1016/j.yebeh.2019. 07.010

13. De Marchis GM, Pugin D, Meyers E, Velasquez A, Suwatcharangkoon S, Park S, Falo MC, Agarwal S, MayerS, Schmidt JM, Connolly ES, Claassen J (2016) Seizure burden in subarachnoid hemorrhage associated with functional and cognitive outcome. Neurology 86(3):253-260. https://doi.org/10. 1212/WNL.0000000000002281

14. Claassen J, Albers D, Schmidt JM, De Marchis GM, Pugin D, Falo CM, Mayer SA, Cremers S, Agarwal $S$, Elkind MS, Connolly ES, Dukic V, Hripcsak G, Badjatia N (2014) Nonconvulsive seizures in subarachnoid hemorrhage link inflammation and outcome. Ann Neurol 75(5):771-781. https://doi. org/10.1002/ana.24166

15. Vespa PM,McArthurDL, XuY, EliseoM, EtchepareM, Dinov I, Alger J, Glenn TP, Hovda D (2010) Nonconvulsive seizures after traumatic brain injury are associated with hippocampal atrophy. Neurology 75(9):792-798. https://doi.org/10. 1212/WNL.0b013e3181f07334

16. Annegers JF, Hauser WA, Lee JR, Rocca WA (1995) Incidence of acute symptomatic seizures in Rochester, Minnesota, 1935-1984. Epilepsia 36(4):327-333. https://doi.org/10.1111/j.15281157.1995.tb01005.x

17. Fink EL, Kochanek PM, Tasker RC, Beca J, Bell MJ, Clark RS, Hutchison J, Vavilala MS, Fabio A, An-

\section{Acute symptomatic seizures and the distinction from status epilepticus on intensive care units in children and adults}

Acute symptomatic seizures are frequently encountered on pediatric and neurological intensive care units. While a seizure semiology allows a diagnosis based on clinical criteria, the diagnosis of electrographic seizures requires precise criteria, which were recently updated and published by the American Clinical Neurophysiology Society (ACNS). New aspects are the description of brief potentially ictal rhythmic discharges (BIRD) and the description of the ictal-interictal continuum (IIC). The interpretation of the electroencephalographic (EEG) criteria is ideally performed by an interdisciplinary approach between neurophysiologists and intensive care physicians. The correlation of increasing seizure burden with dose-dependent neurological deterioration was confirmed in pediatric and neurological studies performed in the respective intensive care units.

\section{Keywords}

Brief potentially ictal rhythmic discharges (BIRD) - Ictal-interictal continuum (IIC) - Seizure burden . Definition · American Clinical Neurophysiology Society (ANCS)

gus DC, Watson RS, Prevalence of Acute critical Neurological disease in children: a Global Epidemiological Assessment (PANGEA) Investigators (2017) International survey of critically ill children with acute neurologic insults: the prevalence of acute critical neurological disease in children: a global epidemiological assessment study. Pediatr Crit Care Med 18(4):330-342. https://doi.org/10. 1097/PCC.0000000000001093

18. Duncan H, Hutchison J, Parshuram CS (2006) The pediatric early warning system score: a severity of illness score to predict urgent medical need in hospitalized children. J Crit Care 21(3):271-278. https://doi.org/10.1016/j.jcrc.2006.06.007

19. Kowalski RL, Lee L, Spaeder MC, Moorman JR, Keim-Malpass J (2021) Accuracy and monitoring of pediatric early warning score (PEWS) scores prior to emergent pediatric intensive care unit (ICU) transfer:retrospective analysis. JMIR Pediatr Parent 4(1):e25991. https://doi.org/10.2196/25991

20. Sahin S, Yazici MU, Ayar G, Karalok ZS, Arhan EP (2016) Seizures in a pediatric intensive care unit: a prospective study. J Trop Pediatr 62(2):94-100. https://doi.org/10.1093/tropej/fmv076 